\title{
Development of a Dengue Vaccine and Its Use in Pregnant Women
}

\author{
Alejandra María Giraldo-García ${ }^{1,2}$ • Jhon Carlos Castaño-Osorio ${ }^{1}$
}

Published online: 23 October 2019

(C) Springer Nature Switzerland AG 2019

\begin{abstract}
Purpose of Review This article discusses dengue virus infection, its effects on mothers and their infants during pregnancy, and the significant issues regarding the development of a safe and effective vaccine against dengue virus for administration to pregnant women.

Recent Findings The risk of pregnant women acquiring dengue virus infection is a rising concern due to the broader geographical distribution of this mosquito-borne flaviviral infection. Several studies have suggested that maternal infection with dengue during pregnancy is associated with a higher risk of severe dengue as well as adverse pregnancy outcomes and maternal and infant mortality. For this reason, both pregnant women as well as non-pregnant women of reproductive age living in endemic areas are important target groups for vaccination against dengue virus. However, similar to many other viral illnesses, there are no current clinical studies on candidate vaccines for dengue infection that include pregnant women as an experimental group.

Summary Dengue virus infection displays a very broad clinical spectrum ranging from asymptomatic infection to non-complex febrile disease and, in a minority of cases, a severe hemorrhagic disease characterized by a life-threatening refractory hypovolemic shock. Several studies suggest that dengue infection during pregnancy is associated with a higher risk of severe dengue and adverse pregnancy outcomes.

Conclusion Despite the risk of obstetric complications in pregnant women due to infection with dengue virus infection, there is currently no specific treatment or licensed vaccine for use during pregnancy.
\end{abstract}

Keywords Flavivirus · Dengue virus · Vaccines · Pregnancy, Perinatal morbidity and mortality · Obstetrical morbidity · Mosquito-borne disease $\cdot$ Maternal morbidity $\cdot$ Global maternal health $\cdot$ CYD-TDV vaccine $\cdot$ Denvaxia $\cdot$ Antibody-dependent enhancement

\section{Introduction}

Dengue virus (DENV) illness is the most important and widespread arthropod-borne viral (arboviral) infection in humans, and is believed to be one of the most top emerging diseases in

This article is part of the Topical Collection on Vaccines in Pregnant Women \& Infants

Jhon Carlos Castaño-Osorio

jhoncarlos@uniquindio.edu.co

1 Grupo de inmunología Molecular, Facultad de Ciencias de la Salud, Universidad del Quindío, Carrera 15 Calle 12 Norte,

Armenia, Quindío, Colombia

2 Programa Licenciatura en Ciencias Naturales, Facultad de Ciencias de la Educación, Universidad del Quindío, Carrera 15 Calle 12 Norte, Armenia, Quindío, Colombia
Asia and Latin America. DENV is transmitted by mosquitos belonging to the genus Aedes that are geographically widespread in both subtropical and tropical regions of the globe. DENV belongs to the Flavivirus genus, which comprises other medically important viruses such as Japanese encephalitis virus (JEV), tick-borne encephalitis virus (TBEV), Kyasanur forest disease (KFD), Omsk hemorrhagic fever (OHF), West Nile virus (WNV), yellow fever virus (YFV), and Zika virus (ZIKV). DENV has a $\sim 11-\mathrm{kb}$ genome that encodes a polyprotein divided into three structural proteins (capsid, premembrane/membrane [prM], and envelope [Env]) and seven non-structural proteins (NS1, NS2A, NS2B, NS3, NS4A, NS4B, and NS5). This virus displays four distinct serotypes, namely DENV-1, DENV-2, DENV-3, and DENV-4 [1]. Persons infected with DENV infection can be asymptomatic, or they can present clinically with dengue fever (DF), dengue with warning signs (DWS+), or severe dengue (SD, also 
known as dengue hemorrhagic fever). A life-threatening form of dengue infection that is characterized by hemorrhage, vascular collapse, and hypotension is termed dengue shock syndrome (DSS) - it tends to affect children under 10 years of age.

Currently, there is an approved vaccine against DENV for use in humans [2]; however, it is not authorized for use during pregnancy. This is attributed to several biological, technical, and bioethical challenges that limit the development of an efficient, cost-effective, and safe vaccine in a mother-baby setting. Among these challenges is the cross-reactivity of the specific immune response, both humoral and cellular, between DENV and other flaviviruses such as ZIKV. Accordingly, to avoid the risk of antibody-dependent enhancement (ADE), a safe vaccine against DENV should confer protective immunity against the four serotypes as well as protect against ZIKV given the overlapping geographic distributions of these viruses and their mosquito vectors [3].

\section{Global Dengue Virus Epidemiology}

Infections by arboviruses are an increasing global public health concern [4]. According to the World Health Organization (WHO), dengue incidence has dramatically increased worldwide in the last decades. It is estimated that 390 million DENV infections occur each year (95\% CI 284-528 million), including 96 million (95\% CI 67-136 million) cases that manifest clinically. Moreover, 3.9 billion people in 128 countries are estimated to be at risk of DENV infection [5]. The recent estimate of 390 million infections annually shows that dengue disease burden has tripled when compared with previous predictions of 50 to 100 million reported cases of dengue without warning signs, and 250,000 to 500,000 patients hospitalized due to DWS+ and SD. Similar to other viral infections occurring in the tropics, these current epidemiological data may be under-reported and not representative of the true prevalence of disease. Also, by 2080, it is estimated that more than 5 to 6 billion people worldwide will be exposed to DENV transmission due to climate change and population growth [6].

The Pan American Health Organization (PAHO) recently issued an alert of a new epidemic outbreak in the region after 2 years of low dengue incidence. Between epidemiologic weeks 1 and 30 of 2019, 2,029,342 cases of dengue were reported in the Americas (an incidence of 207.9 cases per 100,000 inhabitants), including 723 deaths. Among these reported cases, $846,342(42 \%)$ were confirmed by laboratory methods. Also, 12,268 $(0.6 \%)$ cases were classified as SD (with Honduras having the highest proportion) and the mortality was $0.04 \%$ (which was greatest in Guatemala). The number of cases by week $30(2,029,342)$ exceeded the total number of annually reported cases for 2017 and 2018. Meanwhile, the number of reported cases to date is below the historic value registered during the epidemic of 2015-2016 [7]. Similarly, in southern Asia, the Directorate General of Health Services (GDHS) in Bangladesh reports a total of 19,513 cases of dengue as of 2019, including 3464 cases in Dhaka capital district. Among these cases, the deaths of two pregnant women in Dhaka contributed to a total of 48 deaths caused by dengue infection in this country [8].

\section{Clinical Features of Dengue Infection}

Dengue virus infections include a range of well-described clinical illnesses ranging from asymptomatic infection to a self-limiting febrile illness, dengue fever, to severe dengue (shock and death), a clinical syndrome that typically presents with capillary permeability and can lead to dengue shock syndrome and dengue hemorrhagic fever. The atypical presentations of severe dengue are encephalitis, hepatitis, and renal dysfunction [9]. The incubation period of any DENV Infection is from 4 to 8 days, and most patients are able to recover after a self-limiting illness; however, a small proportion develop a severe form of the disease which is mainly characterized by plasma leakage with or without bleeding [10]. On the other hand, in 2$3 \%$ of secondary infections with another serotype, there is a higher risk of increased disease severity, causing lifethreatening Dengue with Warning Signs (DWS+) and Severe Dengue (SD), according to the revised WHO dengue case classification (DENCO) $[11,12]$. Dengue without Warning Signs (DWS-) is seen more often in adults and adolescents and can manifest with only a mild fever only, or a more disabling disease characterized by symptoms occurring mainly in the early febrile stage. These include the sudden onset of high fever, severe headache, retro-orbital pain, myalgia, arthralgia, and rash. In the critical phase, the skin is flushed with the appearance of a petechial rash, occurring predominantly around the time of febrile clinical defervescence, when an increase in capillary permeability accompanied by increased hematocrit can occur. This leads to hypovolemic shock that can result in organ impairment, metabolic acidosis, disseminated intravascular coagulation, and severe hemorrhage. If untreated, mortality can be as high as $20 \%$; but with proper case management and intravenous rehydration, mortality can be reduced to less than $1 \%$ [10]. While SD affects mostly children under 15 years of age, it can also occur in adults, such as in pregnant women. SD causes a transient increase in vascular permeability resulting in plasma leakage with high fever, bleeding, thrombocytopenia, and hemoconcentration, which can lead to shock [5, 12]. Two factors, namely, antibodydependent enhancement (ADE) and inherent virulence of the DENV viruses, seem to be most important in the pathogenesis of the disease [11]. 


\section{Pathophysiology of Dengue Virus Infections}

The pathophysiology of SD is multifactorial due to a complex interaction between the virus and the host-mediated immune response. DENV infection can lead to a dysfunction of the vascular endothelium due to increased permeability, which ultimately causes refractory hypovolemic shock, abnormalities of blood clotting, and eventually thrombocytopenia and ischemia and necrosis of infected cells. Several immunopathological mechanisms, such as ADE, are proposed to contribute to the increased risk of developing DWS+/SD. It is widely acknowledged that antibodies with low avidity can increase the entry of the heterologous virus into the host cells, thus eliciting a hyperimmune reaction that increases vascular permeability and leads to a potentially fatal hypovolemic shock $[5,11,13,14]$. Similarly, a primary infection induces specific memory $\mathrm{T}$ lymphocyte responses against the infecting serotype as well as cross-reactive antibodies against other serotypes, which are activated by viral epitopes expressed on infected cells during a secondary infection by a heterologous DENV. This prompts the production of pro-inflammatory cytokines (TNF $\alpha$, IL6, IL8, IL10, IL12, macrophage migration inhibitory factor, HMGB 1, MCP-1, and matrix metalloproteinases) by $\mathrm{T}$ cells, monocytes, macrophages, and mastocytes, which lead to plasma loss from the vascular endothelium and hypovolemic shock. Meanwhile, the secreted NS1 protein, coupled with anti-NS1 antibodies and complement activation, can stimulate the production of proinflammatory cytokines associated with DWS+/SD, resulting in DENV-induced vascular drainage [15].

Infection with DENV produces long-term or even life-long immunity to reinfection with the same strain of the virus. In endemic regions persons are frequently secondarily reinfected with a heterologous serotype of DENV — this is the most significant risk factor for developing severe infection.

\section{Dengue Infections in Pregnant Women and Their Infants}

The risk of women acquiring DENV during pregnancy is a major concern due to the increasing number of epidemics, broader geographical distribution of disease transmission, and population growth. Several studies suggest that dengue infection during pregnancy is associated with a higher risk of SD and adverse pregnancy outcomes. Despite this, dengue is not often suspected in pregnant women and, consequently, is not diagnosed in a timely manner. A contributing factor is that infection by DENV during pregnancy can be a diagnostic dilemma because of the physiological changes of pregnancy and the obstetric complications found in the clinical practice. For this reason, the diagnosis and management of atypical presentations of DENV are of great importance. A systematic literature review conducted by Pouliot et al. in 2010 considered 30 publications on DENV in pregnant women (19 case reports, 9 case series, and 2 comparative studies), indicating that maternal infection by dengue is a significant risk factor for adverse pregnancy outcomes [16]. Singla et al. [17] reported a $16.1 \%$ frequency of premature births, in addition to $60 \%$ and $28.6 \%$ frequencies of spontaneous abortions in infected mothers occurring during the first and second trimesters, respectively, and a $37.5 \%$ frequency of premature births in the third trimester.

The most common obstetric complication observed by Sharma et al. 2016 in cases of DENV-infected pregnant women in a hospital in New Delhi was oligohydramnios in $43 \%$ of cases. Also, seven women developed bleeding and three women died. Among perinatal complications, there were three intrauterine deaths, six neonatal care unit entries, and one neonatal death. Consequently, DENV infection in pregnancy can be associated with high maternal and perinatal mortalities; thus, early admission and timely disease management in infected pregnant women is of importance [18]. A rigorous control program during pregnancy should be implemented, including early ultrasound examination to detect plasma loss, the presence of ascites, pleural effusion, or oligohydramnios in pregnant women infected with DENV [19].

In a retrospective study of clinical records from 62 pregnant women admitted with DENV infection in a hospital in New Delhi, India, between July and December of 2015, Agarwal et al. found that abortions during the first two trimesters, fetal death and maternal morbidity and mortality were significantly more common among women with DWS+ and SD than in women with dengue without warning signs $(P<0.001)$. Also, the authors found a $41 \%$ incidence of premature births among women diagnosed in the early third trimester. There was a case of fetal death at 37 weeks and no cases of neonatal death. In particular, there were no reports of congenital abnormalities in any of the studies, even when maternal infection occurred in the first trimester. Furthermore, the authors found that the transplacental transfer of IgG showed no harmful effects on neonatal outcomes [20]. Rajagopala et al. reported a case of encephalopathy by DENV in a 28 -year-old woman from India in her 37th week of pregnancy [21]. Ribeiro et al. conducted a histopathological and immunohistochemical study on placental tissues and retained products of conception from 24 patients with confirmed dengue infection during pregnancy. The immunohistochemical analyses were positive for dengue virus in 19 placental and three ovular remnants. Among the microscopic placental findings were signs of hypoxia, choriodecidual infection, deciduitis, villitis, and intervillositis; viral antigens were found by immunohistochemistry in the cytoplasm of trophoblast, chorionic villous stromal cells, and decidua [22]. In contrast to these reports, the results from a meta-analysis conducted by Xiong et al. in 2017 do not suggest that maternal infection by DENV increases the 
risk of adverse pregnancy outcomes based on a RR of 0.96 (95\% CI 0.85 to 1.09) for premature birth, RR of 0.99 (95\% CI 0.87-1.12) for low birth weight, OR of 1.77 (95\% CI 0.993.15) for spontaneous abortion, and RR of 3.42 (95\% CI $0.76-$ 15.49) for fetal death. The authors concluded that the current evidence did not suggest that maternal infection by DENV during pregnancy can increase the risk of premature birth, low birth weight, spontaneous abortion, and fetal death [23•]. However, a study from Mexico by Machain-Williams et al. examining the maternal, fetal, and neonatal outcomes among 82 pregnant women diagnosed with DENV infection provided evidence that SD during pregnancy was associated with a high rate of fetal distress and morbidity, emergency cesarean sections, obstetrical hemorrhage, and maternal mortality. Among those mothers having nonsevere dengue, there was no association with maternal mortality, fetal distress, or adverse neonatal outcomes [24••].

In addition to these reports, Nunes et al. reported the case of a 36-year-old Brazilian woman with 29 weeks of pregnancy who manifested severe complications due to infection by DENV, which led to maternal and fetal death. The postmortem analysis of the fetal organs based on RT-PCR and immunohistochemisty using antibodies to the NS3 DENV protein showed the presence of DENV in the brain, placenta, and several fetal organs such as the liver, lungs, and spleen. The histological analyses of the placenta revealed chronic villitis, decidual and villous hemorrhage, edema, and necrosis. The liver was highly abnormal, showing a diffuse area of necrotic hepatocytes with a mononuclear infiltrate, steatosis, hyperplasia of Kupffer cells, a discrete region of lymphocytic infiltration in the sinusoidal capillary, thickening of the endothelium in the central vein, and the presence of edema. In addition, there were signs of fetal tissue dysfunction, such as microglial inflammation, steatosis, arteriolar hyalinosis, inflammatory cells in the alveolar septa, and lymphoid follicular disorder, an increase in cellularity (macrophages, Hofbauer cells, and TCD8 + lymphocytes) and the production of inflammatory mediators, such as IFN- $\alpha$, TNF- $\gamma$, RANTES/CCL5, MCP1/CCL2, and VEGF/R2, in the liver, lungs, spleen, brain, and placenta associated with inflammation of the peripheral tissue of the placenta and fetus. According to these authors, maternal infection by DENV promotes the production of vascular mediators that can alter vascular permeability and facilitate viral entry and tissue and placental barrier dysfunction [25••].

Considering the literature reports and the possible consequences of DENV infection during pregnancy, pregnant women and women of reproductive age living in endemic areas should be vaccinated against DENV and other flaviviruses (Fig. 1) [26, 27].

\section{Development of Vaccines Against Dengue for Use in Pregnant Women}

An important aim of a vaccine against DENV administered during pregnancy will be to prevent the infection of the mother and fetus, the development of DWS+ and SD in the mother, abortion and fetal damage. The vaccine should be a tool to control DENV outbreaks in the short term. DENV was isolated over 70 years ago, yet, to date, there is no specific treatment for dengue disease and the CYD-TDV vaccine approved for use in humans is not authorized for use during pregnancy.

There are multiple obstacles that have delayed the progress in obtaining a DENV vaccine for use during pregnancy. These include [1] the co-circulation of multiple DENV serotypes as well as other flaviviruses; [2] imperfect knowledge of viral pathogenesis; [3] lack of understanding of the immunological mechanisms of the cross-reactive specific cellular and humoral immune responses of DENV antigens with other flaviviruses; [4] lack of a reliable animal model; [5] the complexity of the host immune mechanisms; [6] deficiency of
Fig. 1 Findings of dengue infection during pregnancy
FINDINGS IN PREGNANT WOMEN

- Vomiting, fever, rash, joint
\& muscle pain, headache
- Dengue with alarm signs
- Severe dengue
- Shock
- Thrombocytopenia
- Hemorrhage
- Hypovolemia
- Encephalopathy
- Fetal monitoring
abnormalities
- Oligohydramnios
- Premature delivery
- Maternal death
References[18, 21, 25]

FINDINGS IN THE FETUS AND PLACENTA

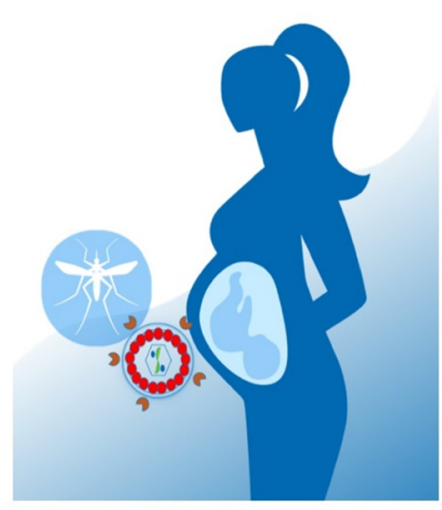

- Placental pathology: deciduitis, choriodeciduitis, villitis, villous necrosis, intervillositis

- Viral antigens detectable in fetal organs and placental tissues (trophoblast, villous stroma, and decidua)

- Inflammatory mediators in liver, lungs, spleen, brain and placenta including INF $\propto$, TNF- $\gamma$, RANTES / CCL5, MCP1 / CCL2, VEGF / R2

- Spontaneous abortion during 1st and 2nd trimesters

- Stillbirth

- Neonatal death

References [17, 18, 20, 22, 24, 25] 
clinical studies involving pregnant women and their infants; [7] knowledge gaps of the immune mechanisms of DENV infection during pregnancy and in the fetus since pregnancy can alter the immune response and the fetal immune response can change throughout pregnancy. Additional challenges to developing an efficacious dengue vaccine for use in pregnant women are [1] the vaccine must protect the developing fetus and it must achieve protective immunity before the moment of maximum infection vulnerability; [2] many women are unaware of their pregnancies until late in the first trimester; [3] vaccines with live attenuated viruses can be protective after a single dose, yet these may not be suitable candidates for administration during pregnancy; and [4] inactivated subunit recombinant vaccines and other non-replicative vaccines that are more suitable for use during pregnancy generally require multiple doses to achieve protective immunity, which delays the establishment of an effective immunity beyond the window of maximum vulnerability of the fetus $[6,27]$.

In December 2015 a live, attenuated tetravalent vaccine named CYD-TDV or Denvaxia (Sanofi Pasteur) was initially approved in Mexico for individuals aged 9 years and older, which has since been used in several endemic Asian and Latin American countries for protection against the four viral serotypes. In pre-clinical studies the CYD-TDV vaccine did not demonstrate any teratogenicity in the progeny of vaccinated pregnant animals [26]. Although the CYD-TDV vaccine has shown an adequate general safety and efficiency profile in the clinical trials, some key research aspects remain, such as the frequency of very rare adverse events after immunization, the duration of the induced protection, and its effectiveness when used in public health programs. A safety-related question regarding this vaccine is whether it is safe for use in special risk groups, such as immunocompromised persons and pregnant/ breastfeeding women [28]. The knowledge gaps on the use of this vaccine in early pregnancy and its safety have hindered the development and recommendation of an effective vaccine against DENV and other flaviviruses in pregnant mothers since maternal immunization is a challenge to vaccinology [27, 29-31]. Although the CYD-TDV vaccine, like many other live virus vaccines, has been contraindicated for administration during pregnancy due to the theoretical possibility of risk to the fetus, there were pregnant women who inadvertently received the vaccine during the clinical development of Denvaxia [26]. There were 58 women identified who were considered exposed to CYD-TDV during the pre-specified risk window for pregnancy among all persons enrolled in the clinical trials. Of these 58 women, 22 were sub-categorized as "exposed and pregnant," i.e., vaccinated with CYD-TDV during the interval starting from last menstrual period (LMP) + 7 days, and 36 were "exposed and not pregnant," i.e., vaccinated in the period between 30 days before and 7 days after their LMP. Follow-up and analysis of the clinical course of the pregnancies and health of their newborn infants demonstrated that there were no adverse pregnancy outcomes among the young women who were exposed to Denvaxia during early pregnancy compared with controls [26].

In order to address issues in the development of a vaccine against DENV, it is important to critically assess the safety of the vaccine during pregnancy in terms of determining the substantial risk of infection for the mother and the fetus in the absence of immunization. Besides, considerations must be taken regarding the possible immunopathological complications caused by ADE due to previous infections by one of the DENV serotypes or other prevalent flaviviruses, such as ZIKV. In this context, Fowler et al. [32] showed that mice born with maternal anti-ZIKV antibodies develop SD and viremia following experimental infection with DENV compared with mice from non-immune mothers. Likewise, the previous immunization with licensed vaccines for other flaviviruses, such as JEV and YFV, could also pose a risk for vaccination against DENV.

The routine administration of live vaccines in pregnant women is generally contraindicated due to concerns of fetal damage derived from attenuated infectious agents. However, not all live vaccines can lead to this problem. The greatest concern is for those live vaccines that replicate systemically and can cross the placental barrier. Despite the accidental exposure during pregnancy to several types of live vaccines (e.g., vaccines against rubella, yellow fever, and smallpox) in hundreds or thousands of women, there is convincing evidence of fetal damage only for the vaccine against smallpoxa small increase in the risk of birth defects $(2.4 \%$ compared with $1.5 \%$ ) among women vaccinated in the first trimester. Furthermore, in a retrospective study, Tsai et al. reported the first case of congenital infection with the vaccine strain of the yellow fever virus (YFV) after immunization of pregnant women who were unaware of their state and were vaccinated during a massive YFV immunization campaign in Trinidad. The authors found that 1 of 41 children born to these women showed IgM and neutralizing antibodies against the virus, indicating congenital infection. Although the pregnancies did not show any complications and the newborns were born at full term and healthy, the neurotropism of YFV in the developing nervous system and the reported possibility of transplacental infection must be considered. This underlies the warning to avoid YFV vaccination during pregnancy [33]. Similarly, Nishioka et al. reported the possible association of the accidental administration of the YFV vaccine during early pregnancy and the occurrence of spontaneous abortion with an OR (relative risk estimate) of 2.29 (95\% CI 0.65-8.03).

Based on these findings, a sensible recommendation is to avoid YFV vaccination in pregnant women unless the risk of acquiring YFV exceeds the risk of abortion in relation to the vaccine [34]. For this reason, vaccines against yellow fever and smallpox are offered only to women with a high risk of infection since the potential benefits greatly exceed the risks. 
To guarantee that pregnant women have access to vaccines with reassuring safety data, the vaccine candidates with the highest probabilities of safe administration during pregnancy must be assessed [27]. Despite the precaution of not administrating live vaccines in pregnant women, there are safety data for some attenuated vaccines against flaviviruses that were inadvertently administered to women in the very early stages of pregnancy who were unaware of their condition as previously discussed for Denvaxia.

\section{Considerations of Previous Immunity Against Other Flaviviruses in the Development of a Vaccine Against Dengue Virus}

Published studies have demonstrated that antibodies isolated from patients infected with DENV or ZIKV have high crossreactivity or cross-protection both in vitro and in vivo [35]. Therefore, ZIKV can be considered a fifth member of the DENV serocomplex, a factor that should be accounted for in the vaccine development approaches of the two viruses [36]. Lima et al. conducted a study on non-human primates infected with ZIKV, finding that pre-existing immunity to DENV or YFV leads to greater activation of T-CD4 cells and higher titers of anti-ZIKV IgG. This indicates that the specific responses to ZIKV benefit from the pre-existing immune memory for other flaviviruses. However, this pre-immunity against flaviviruses did not provide cross-protection against infection by ZIKV [37]. Zhao et al. found that some anti-ZIKV antibodies produced during a natural infection facilitated DENV infection in vitro. Therefore, it is necessary to determine the implication of pre-existing antibodies in pregnant women in areas with a high prevalence of DENV, ZIKV, and other flaviviruses on the effectiveness and safety of a vaccine for use during pregnancy [38, 39].

\section{Bioethical Considerations for the Development of Dengue Virus Vaccines for Use in Pregnant Women}

Historically, pregnant and breastfeeding women and their offspring have been largely excluded from research agendas, experimental drug and vaccine design protocols, clinical trials, and vaccine investment strategies against epidemic threats, which can also lead to their exclusion from future vaccination campaigns during infectious disease outbreaks, such as DENV [24••, 27, 28]. This is unfair to pregnant women and their offspring - it constitutes an important public health as well as human rights issue due to the risk of exposure of pregnant women in areas with a high prevalence of transmissible and, in some cases, life-threatening infectious diseases.
To guarantee that the needs of pregnant women and their babies are justly addressed, a new approach to research goals and the development of vaccines and their administration that are inclusive of pregnant women are needed. Most drugs available in the market have little or no information regarding their safe and effective use during pregnancy. This is unacceptable; therefore, a paradigm shift is required to safely and responsibly include pregnant women in research so that both they and their offspring can eventually benefit from critical interventions to support their health and well-being [27, 30, 40]. For this, vaccine clinical trials should include pregnant women in different stages of pregnancy from areas with active disease transmission.

\section{Conclusion}

Despite the risk of maternal and fetal complications due to DENV infection during pregnancy, there are currently no specific treatments or licensed vaccines for use in mothers and their infants. Although several vaccines against DENV are currently undergoing phase II and phase III clinical trials, pregnant and lactating women have not been included in these investigations. This is unfortunate - the risk of obstetric and perinatal complications for pregnant women due to DENV in endemic areas places these women and their infants at heightened risk for morbidity and mortality from infection. Researchers must reconsider their historical policies of exclusion, and incorporate pregnant women in the design, clinical trials, and administration of DENV vaccines to effectively prevent maternal and fetal complications of DENV infection.

\section{Compliance with Ethical Standards}

Conflict of Interest The authors declare that they have no conflict of interests.

Human and Animal Rights and Informed Consent This article does not contain any studies with human or animal subjects performed by any of the authors.

\section{References}

Papers of particular interest, published recently, have been highlighted as:

- Of importance

- Of major importance

1. Dowd KA, Demaso CR, Pelc RS, Speer SD, Alexander R, Smith Y, et al. Broadly neutralizing activity of Zika virus-immune sera identifies a single viral serotype. Cell Rep. 2016;16(6):1485-91. 
2. Li G, Teleki C, Wang T. Memory T cells in flavivirus vaccination. Vaccines. 2018;6(4):pii: E73 Available from: https://www.ncbi. nlm.nih.gov/pmc/articles/PMC6313919/.

3. Harrison SC. Immunogenic cross-talk between dengue and Zika viruses. Nat Immunol. 2016;17(9):1010-2. Available from:. https://doi.org/10.1038/ni.3539.

4. Fritzell C, Rousset D, Adde A, Kazanji M, Van Kerkhove MD, Flamand C. Current challenges and implications for dengue, chikungunya and Zika seroprevalence studies worldwide : a scoping review. PLoS Negl Trop Dis. 2018;12(7):e0006533 Available from: https://www.ncbi.nlm.nih.gov/pmc/articles/PMC6062120/.

5. WHO. Dengue and severe dengue. WHO Fact Sheet. 2012. p. 1-4. Available from: www.who.int/mediacentre/factsheets/fs117/en/ index.html

6. Pang EL, Loh HS. Towards development of a universal dengue vaccine - how close are we? Asian Pac J Trop Med. 2017;10(3): 220-8. Available from:. https://doi.org/10.1016/j.apjtm.2017.03. 003.

7. PAHO. Actualización Epidemiológica Dengue. 9 de Agosto de 2019. Available from: https://www.paho.org/hq/index.php? option=com_docman\&view=download\&category_slug=dengue2158\&alias=49621-9-de-agosto-de-2019-dengue-actualizacionepidemiologica-1\&Itemid=270\&lang=es

8. The Daily Star. 2 pregnant women die of dengue at BSMMU. August 2, 2019. Available from: https:/www.thedailystar.net/city/ news/2-pregnant-women-die-dengue-bsmmu-1780840

9. Webster DP, Farrar J, Rowland-Jones S. Progress towards a dengue vaccine. Lancet Infect Dis. 2009;9(11):678-87 Available from: http:/linkinghub.elsevier.com/retrieve/pii/S1473309909702543.

10. Guzman MG, Harris E. Dengue. Dengue. Lancet. 2015;385(9966): 453-65. https://doi.org/10.1016/S0140-6736(14)60572-9.

11. Swaminathan S, Khanna N. Dengue: recent advances in biology and current status of translational research. Curr Mol Med. 2009;9(2):152-73.

12. Hadinegoro SRS. The revised WHO dengue case classification: does the system need to be modified? Paediatr Int Child Health. 2012;32(sup1):33-8.

13. Morens DM. Antibody-dependent enhancement of infection and the pathogenesis of viral disease. Clin Infect Dis. 1994;19(3):500 12.

14. Morens D, Halstead SB. Measurement of antibody-dependent infection enhancement of four dengue virus serotypes by monoclonal and polyclonal antibodies. J Gen Virol. 1990;71(Pt 12): 2909-14.

15. Hober D, Poli L, Roblin B, Gestas P, Chungue E, Granic G, et al. Serum levels of tumor necrosis factor-alpha (TNF-alpha), interleukin-6 (IL-6), and interleukin-1 beta (IL-1 beta) in dengueinfected patients. Am J Trop Med Hyg March. 1993;48:324-31.

16. Pouliot SH, Xiong X, Harville E, Paz-Soldan V, Tomashek KM, Breart $\mathrm{G}$, et al. Maternal dengue and pregnancy outcomes: a systematic review. Obstet Gynecol Surv. 2010;65(2):107-18.

17. Singla N, Arora S, Goel P, Chander J, Huria A. Dengue in pregnancy: an under-reported illness, with special reference to other existing co-infections. Asian Pac J Trop Med. 2015;8(3):206-8.

18. Sharma S, Jain S, Rajaram S. Spectrum of maternofetal outcomes during dengue infection in pregnancy: an insight. Infect Dis Obstet Gynecol. 2016;2016:1-4 Available from: http:/www.hindawi.com/ journals/idog/2016/5046091/.

19. Kularatne SAM. Dengue fever. BMJ. 2015;351:h4661. https://doi. org/10.1136/bmj.h4661.

20. Agarwal K, Malik S, Mittal P. A retrospective analysis of the symptoms and course of dengue infection during pregnancy. Int $\mathrm{J}$ Gynecol Obstet. 2017;139(1):4-8.

21. Rajagopala L, Satharasinghe RL, Karunarathna M. A rare case of dengue encephalopathy complicating a term pregnancy. BMC Res Notes. 2017;10(1):79. https://doi.org/10.1186/s13104-017-2391-5
Available from: https://www.ncbi.nlm.nih.gov/pmc/articles/ PMC5288850/.

22. Ribeiro CF, Lopes VGS, Brasil P, Pires ARC, Rohloff R, Nogueira RMR. Dengue infection in pregnancy and its impact on the placenta. Int J Infect Dis. 2017;55:109-12. Available from:. https://doi. org/10.1016/j.ijid.2017.01.002.

23. Xiong YQ, Mo Y, Shi TL, Zhu L, Chen Q. Dengue virus infection during pregnancy increased the risk of adverse fetal outcomes? An updated meta-analysis. J Clin Virol. 2017;94:42-49. Available from: https://doi.org/10.1016/j.jcv.2017.07.008. In this article the authors conclude that the current evidence did not suggest that maternal infection by DENV during pregnancy can increase the risk of premature birth.

24.• MacHain-Williams C, Raga E, Baak-Baak CM, Kiem S, Blitvich BJ, Ramos C. Maternal, fetal, and neonatal outcomes in pregnant dengue patients in Mexico. Biomed Res Int. 2018;2018. doi: https:// doi.org/10.1155/2018/9643083. Availaboe from: https://www.ncbi. nlm.nih.gov/pmc/articles/PMC5828467/. In this article the authors provide evidence that SD during pregnancy is associated with a high rate of fetal distress, emergency cesarean sections, and maternal mortality.

25.• Nunes P, Nogueira R, Coelho J, Rodrigues F, Salomão N, José C, et al. A stillborn multiple organs' investigation from a maternal denv-4 infection: histopathological and inflammatory mediators characterization. Viruses. 2019;11(4):319 Available from: . https://www.ncbi.nlm.nih.gov/pmc/articles/ PMC6521294/. In this article, the authors describe the histopathological and molecular findings from the placenta and fetal organs from a case of congenital dengue that resuted in stillbirth.

26. Skipetrova A, Wartel TA, Gailhardou S. Dengue vaccination during pregnancy - an overview of clinical trials data. Vaccine. 2018 May;36(23):3345-50 Available from: http://linkinghub.elsevier. com/retrieve/pii/S0264410X18305474.

27. Krubiner CB, Faden RR, Karron RA, Little MO, Lyerly AD, Abramson JS, et al. Pregnant women \& vaccines against emerging epidemic threats : ethics guidance for preparedness , research, and response. Vaccine. 2019. pii: S0264-410X(19)30045-3. https://doi. org/10.1016/j.vaccine.2019.01.011. Available from: https://www. sciencedirect.com/science/article/pii/S0264410X19300453?via\% 3Dihub

28. Wichmann O, Vannice K, Asturias EJ, de Albuquerque Luna EJ, Longini I, Lopez AL, et al. Live-attenuated tetravalent dengue vaccines: the needs and challenges of post-licensure evaluation of vaccine safety and effectiveness. Vaccine. 2017;35(42. Available from: ):5535-42. https://doi.org/10.1016/j.vaccine.2017.08.066.

29. Pagliusi S, Dennehy M, Kim H, DCVMN AGM Organizing Committee. Vaccines, inspiring innovation in health. Vaccine. 2018;36(48):7430-7437. Available from: https://doi.org/10.1016/ j.vaccine.2018.05.035

30. Faden RR, Krubiner CB, Lyerly AD, Little MO, August A, Beigi $\mathrm{RH}$, et al. Ethics, pregnancy, and ZIKV vaccine research \& development The ethics working group on ZIKV research and pregnancy. Vaccine. 2017;35(49):6819-6822. Available from: https://doi. org/10.1016/j.vaccine.2017.09.065

31. Schwartz DA. Clinical trials and administration of Zika virus vaccine in pregnant women: lessons (that should have been) learned from excluding immunization with the Ebola vaccine during pregnancy and lactation. Vaccines. 2018;6(4):81 Available from: https:// www.ncbi.nlm.nih.gov/pmc/articles/PMC6313913/.

32. Fowler AM, Tang WW, Young MP, Mamidi A, Viramontes KM, McCauley MD, et al. Maternally acquired Zika antibodies enhance dengue disease severity in mice. Cell Host Microbe. 2018;24(5): 743-750.e5. Available from. https://doi.org/10.1016/j.chom.2018. 09.015 . 
33. Tsai TF, Paul R, Lynberg MC, Letson GW, Tsai TF, Paul R, et al. Congenital yellow fever virus infection after immunization in pregnancy. J Infect Dis. 1993;168(6):1520-3.

34. Nishioka SA, Pires WP, Silva FA, Costa HL. Yellow fever vaccination during pregnancy and spontaneous abortion: A case-control study. Trop Med Int Health. 1998;3(1):29-33.

35. Long F, Doyle M, Fernandez E, Miller AS, Klose T, Sevvana M, et al. Structural basis of a potent human monoclonal antibody against Zika virus targeting a quaternary epitope. Proc Natl Acad Sci USA. 2019;116(5):1591-1596. Available from. http://www. ncbi.nlm.nih.gov/pubmed/30642974.

36. Dejnirattisai W, Supasa P, Wongwiwat W, Rouvinski A, BarbaSpaeth G, Duangchinda T, et al. Dengue virus sero-crossreactivity drives antibody-dependent enhancement of infection with zika virus. Nat Immunol. 2016 Jun 23;17:1102. Available from:-8. https://doi.org/10.1038/ni.3515.

37. Lima NS, Rolland M, Modjarrad K, Trautmann L. T cell immunity and Zika virus vaccine development. Trends Immunol. 2017;38(8): 594-605. https://doi.org/10.1016/j.it.2017.05.004.
38. Zhao H, Fernandez E, Dowd KA, Speer SD, Platt DJ, Gorman MJ, et al. Structural basis of Zika virus-specific antibody protection. Cell. 2016;166(4):1016-27. Available from:. https://doi.org/10. 1016/j.cell.2016.07.020.

39. Silva JVJ Jr, Lopes TRR, Oliveira-Filho EF De, Oliveira RAS, Durães-Carvalho R, Gil LHVG. Current status, challenges and perspectives in the development of vaccines against yellow fever, dengue, Zika and chikungunya viruses. Acta Trop. 2018;182:257-263. Available from: https://doi.org/10.1016/j.actatropica.2018.03.009

40. Kochhar S, Edwards KM, Maria A, Alvarez R, Moro PL, Ortiz JR. Introduction of new vaccines for immunization in pregnancy - programmatic, regulatory, safety and ethical considerations. Vaccine. 2019;37(25):3267-77. https://doi.org/10.1016/j.vaccine.2019.04. 075 .

Publisher's Note Springer Nature remains neutral with regard to jurisdictional claims in published maps and institutional affiliations. 